\title{
Lima festeja a la Inmaculada (1656): texto criollo y poderes urbanos ${ }^{*}$
}

\section{Lima Celebrates the Immaculate Conception (1656): Creole Text and City Powers}

PILAR LATASA

Universidad de Navarra

platasa@unav.es

\section{RESUMEN}

Este articulo analiza las fiestas a la Inmaculada que se celebraron en Lima en 1656 a partir de la relación festiva escrita por Diego de León Pinelo Gutiérrez. Este texto criollo reinterpreta en clave barroca la devoción inmaculista de la ciudad de Los Reyes. A través de él se estudian los prolongados festejos que contaron con la participación de las instituciones y corporaciones de poder más representativas de la ciudad y movilizaron considerables recursos y personas. Asi, esta relación se concibe como una exaltación politica y religiosa de la ciudad de Lima, propia del género festivo y de la literatura corográfica, que se conecta también con el naciente discurso criollo.

Palabras clave: fiestas; Inmaculada; Diego de León Pinelo; Universidad de San Marcos; Cabildo de Lima

* Este trabajo se ha desarrollado dentro del proyecto: Discurso y poder, lengua y autoridad en el mundo hispánico (s. XVI-XVII). Ministerio de Economía y Competitividad, Programa Nacional de Promoción General del Conocimiento (Área de Historia y Arte). Ref.: HAR2012-31536. 


\section{ABSTRACT}

Analysis of the festivities of the Immaculate Conception celebrated in Lima in 1656, from the account written by Diego de León Pinelo Gutiérrez. This Creole text reinterprets in baroque terms the cult of the Immaculate Conception in the City of Kings. Through it we can study these lengthy festivals, which involved the participation of the city's most important institutions and corporate bodies, mobilizing considerable resources and people. Thus, the text is conceived as a political and religious exaltation of the city of Lima, typical of the festive genre and of the chorographic literature, which also connects with the emerging Creole discourse.

Keywords: festivities; Immaculate Conception; Diego de León Pinelo; University of San Marcos; Lima Cabildo

En el año 1656 la Universidad de San Marcos de Lima protagonizó
una mascarada en honor de la Inmaculada Concepción que apenas ha sido trabajada hasta el momento, a pesar de que existe una relación impresa de la misma, la compuesta por Diego de León Pinelo. ${ }^{1}$ En este artículo, en primer lugar, se situarán estas celebraciones en relación con los ciclos festivos inmaculistas que tuvieron lugar dentro de la monarquía hispánica. En segundo lugar, se abordará el análisis del texto dentro de su género, con especial atención a su autor, miembro de la destacada familia criolla de los León Pinelo. Finalmente, en tercer lugar, se establecerá el calendario de las fiestas y sus protagonistas con el fin de situar la mascarada en su entorno inmediato.

\section{LA INMACULADA Y LIMA}

Para enmarcar esta celebración en su contexto histórico es preciso retrotraerse a 1615, año en que Felipe III estableció en la corte madrileńa la Real Junta de la Inmaculada y envió a Roma la embajada

1 León Pinelo Gutiérrez s.f. La única noticia que hasta hace poco se tenía de estas fiestas procedía de los datos que aportan Mugaburu y Mugaburu (1917, I: 38-42). Los estudios de Vargas Ugarte (1956: 129), Bradley (1992: 12-13) y Durán Montero (1994: 194-195) se apoyaron en dicho estudio. Por otro lado, la relación inédita fue trabajada por primera vez por Mujica Pinilla (1999: 191-219). 
presidida por el benedictino Plácido Tosantos, con el fin de promover la declaración dogmática del misterio tras las fuertes controversias suscitadas en la ciudad de Sevilla entre 1613 y 1615. Aunque con estas medidas no se consiguió la deseada exposición del dogma, sí se propició la aprobación de una declaración del 31 de agosto de 1617 - en tiempos del papa Paulo V (1605-1621) — por la que se permitía a los inmaculistas mantener su parecer en público. ${ }^{2}$

La disposición papal fue recibida con regocijo y provocó el primer gran ciclo festivo inmaculista. ${ }^{3}$ Así, desde Andalucía, las celebraciones se extendieron por todo el mundo hispánico y, en la capital del virreinato peruano, se llevaron a cabo las solemnidades de 1617 y $1618 .{ }^{4} \mathrm{En}$ la primera, tras la misa solemne en la catedral presidida por el arzobispo Bartolomé Lobo Guerrero, el virrey junto con los tribunales, el Cabildo y la Universidad juraron y votaron defender «la opinión piadosa»; la celebración en las calles corrió a cargo de la Universidad que organizó una mascarada. ${ }^{5}$ De la segunda se conserva una interesante relación firmada por Diego Cano, capellán de la Universidad, que confirma la conexión existente entre los festejos peninsulares y americanos: «Tres años a que vienen a estos reinos de los de España y corte de vuestra majestad, traen nuevas y relaciones de fiestas en que se ha celebrado la Inmaculada Concepción de la Virgen Nuestra Señora y, últimamente, las que se hicieron a la bula tan favorable». ${ }^{6}$

En la década siguiente, ya durante el reinado de Felipe IV, se dio un nuevo paso con el decreto «Sanctisssimus» de Gregorio XV, del 2 de junio de 1622, por el cual, aunque se daba libertad para defender las teorías maculistas o las inmaculistas, se privilegiaban estas últimas al prohibirse

2 Gutiérrez 1955: 18-20.

3 Algunos autores lo denominan también «el gran ciclo celebrativo sevillano de 1617». Entre otras, cabe destacar las fiestas de Ronda (1615), Jerez (1616), Sevilla (1617), Córdoba (1617), Salamanca (1618), Baeza (1618), Utrera (1618) y Pamplona (1618). Al respecto, véase García Bernal 2006a: 102-110.

4 Eguiguren 1940-1951, II: 613-621 y 621-656.

5 Vargas Ugarte 1956, I: 127-129.

6 Cano Gutierrez 1619; énfasis agregado. 
la defensa pública o privada de opiniones maculistas. ${ }^{7}$ La noticia dio lugar a un nuevo ciclo festivo hispánico en el que, coincidiendo con las grandes funciones públicas que tuvieron lugar por la canonización de los cinco santos españoles en 1622, se asentó la simbología festiva barroca iniciada en el ciclo inmaculista anterior. Este nuevo ciclo tuvo una dimensión más supranacional y coincidió además con el éxito del impreso festivo. ${ }^{8}$ Sin embargo, no tuvo eco en la ciudad de Los Reyes, tal vez por la cercanía de las grandes celebraciones de 1617 y 1618, antes mencionadas.

La actividad española a favor de la causa inmaculista se reavivó en 1642, a partir de un decreto de Urbano VIII en el que establecía el nuevo elenco de fiestas de precepto, que no incluía la Inmaculada: las fiestas no catalogadas debían suprimirse aunque se vinieran celebrando. En España, pronto se encontró una vía para transgredir lo dispuesto: aprovechar la posibilidad de celebrar bajo precepto, además de las fiestas señaladas, la de uno de los patronos principales. ${ }^{9}$ Así, una tras otra, las poblaciones espańolas fueron proclamando o reiterando el patronato concepcionista. A pesar del éxito, se acudió a Roma en búsqueda de una solución menos precaria y, en 1645, se consiguió que Inocencio X declarase el restablecimiento de la Inmaculada como fiesta de precepto «in regnis Hispaniarum». ${ }^{10}$

La autorización coincidió en España con una nueva etapa de fervor concepcionista alentada también por la reacción ante un decreto del Santo Oficio del 20 de enero de 1644 por el que se prohibía utilizar la denominación de Inmaculada. En este nuevo ciclo participaron teólogos, apologistas, las cortes y el pueblo, y en algunos lugares llegaron

7 Gutiérrez 1955: 25-28; y Cortés Peña 2003: 412-414.

8 García Bernal 2006b: 80. Para las de Barcelona, véase Cordón 1999.

9 La elección de patronos había surgido de forma espontánea en los siglos XIV y XV. Urbano VIII estableció, por decreto del 23 de marzo de 1630, que la elección del patrón de un lugar hecha por el pueblo con el consentimiento del obispo debía ser confirmada por la Santa Sede (Gámez Martín 2008: 185).

${ }^{10}$ Gutiérrez 1955: 29-32. Sin embargo, el patronato general de la Inmaculada para los reinos de España e Indias no se consiguió hasta el breve Quantum Ornamenti de 1760 (Gámez Martín 2008: 187). 
a producirse algaradas y disturbios. El rey Felipe IV cursó en 1650 una circular a todos los obispos, iglesias y cabildos para que apoyasen las negociaciones entre Inocencio X y Gonzalo de Castilla, duque del Infantado, que, de nuevo, no tuvieron el éxito esperado. ${ }^{11}$ Sin embargo, al poco tiempo se produjo la elección del nuevo papa, Alejandro VII, y uno de sus primeros actos de gobierno fue la emisión de la bula, del 15 de mayo de 1655 , por la que se restituía el libre uso del apelativo Inmaculada. ${ }^{12}$

Es en el marco de este entusiasmo renovado en defensa del dogma inmaculista en el que se sitúan los festejos que son objeto de este trabajo. La bula por la que Inocencio X restableció la fiesta de la Inmaculada «in regnis Hispaniarum» no incluía los territorios indianos. En Lima, sin embargo, el arzobispo Pedro de Villagómez y Vivanco había accedido en 1654 - a instancias del Cabildo secular - a instaurar la fiesta de precepto y el juramento de la pía doctrina. ${ }^{13} \mathrm{El} 8$ de diciembre de ese año, el día de la Inmaculada, se celebró en la ciudad de Los Reyes con toda solemnidad: tras las misa pontifical en la que predicó Gonzalo de Herrera, provincial de los franciscanos, el virrey Conde de Salvatierra, la Audiencia y los cabildos "votaron todos de guardar y defender la pura y limpia concepción de Nuestra Señora, sin pecado original». Por la tarde, una procesión presidida por una imagen de la Inmaculada recorrió la Plaza Mayor. Finalmente, la ciudad votó que se la nombrara patrona de Lima. ${ }^{14}$

$\mathrm{Al}$ año siguiente, la fiesta se celebró con idéntico ritual. ${ }^{15} \mathrm{La}$ solicitud formal a Roma del patronazgo de la Inmaculada se materializó en agosto de 1656, a través de Gabriel Hernández de Zúñiga y Madrigal, procurador de la ciudad de Los Reyes en la corte madrileña. Este fue

${ }^{11}$ Gutiérrez 1955: 32-41.

12 Cortés Peña 2003: 422-423.

${ }^{13}$ Villagómez y Vivanco pasó al Perú el 7 de marzo de 1632 como obispo electo de Arequipa (Archivo General de Indias [en adelante AGI], Contratación 5.413, n. ${ }^{\circ}$ ) y posteriormente fue promovido a la sede de Lima, en la que estuvo treinta años hasta su fallecimiento el 12 de mayo de 1671 (Vargas Ugarte 1959-1962, III: 1-34). La solicitud se hizo el 4 de diciembre de 1654 (Bermúdez 2001: 99-104).

${ }^{14}$ Mugaburu y Mugaburu 1917, I: 30-31.

${ }^{15}$ Ib.: 36 . 
el encargado de presentar la petición al Pontífice, que se debe entender también en relación con la mencionada bula del 15 de mayo de 1655 . De esta manera, a comienzos de septiembre de 1656, llegó a Lima la noticia de la concesión, que dio lugar a las demostraciones festivas que aquí se estudian. ${ }^{16}$

\section{EL TEXTO Y SU AUTOR}

La obra analizada pertenece al género de relaciones de fiestas. En cierto modo, la relación escrita de los festejos constituía el broche de oro de las celebraciones. Como es habitual en este tipo de relatos, no se trata de una mera descripción de los acontecimientos sino de una recreación literaria que intentaba deslumbrar al lector, hacerle que «despierte la memoria de ellas» y disfrute con todos los pormenores. ${ }^{17}$ Así, aunque el narrador abunda en detalles, se excusa de no ser capaz de recogerlos todos: «No cabe en rasgos breves todo lo que ministra aquí la memoria al genio del que escribe, tan sin ocio en sus ocupaciones, que no le tiene para cultivar el aparato [...]. Sea la pluma delgado pincel que dote los perfiles, no carbón tosco que abrume y canse las ideas». ${ }^{18}$

La narración pretendía afirmar la tradición inmaculista de la institución, según señala su autor: «dejando acreditada la devoción de la Universidad en [las] fiestas del misterio, de suerte que si esta [fiesta] no se aventajó a todas, ninguna la excedió de cuantas se han hecho hasta aquí, y pocas [la] han igualado». ${ }^{19}$ En definitiva se trataba de mostrar la devoción de la ciudad y la Universidad a la Inmaculada tras la concesión del patronazgo por parte de la Santa Sede. Por eso, aunque el objetivo principal era narrar la mascarada que protagonizó la academia — «llave de oro» de las celebraciones y, por su «disposición, lustre, riqueza y pompa», la primera entre ellas-, se relataban también las que protagonizaron

${ }^{16}$ Bermúdez 2001: 107; Vargas Ugarte 1956, I: 129; y Acta del Cabildo del 31 de agosto de 1656, Archivo Histórico de la Municipalidad de Lima [en adelante AHML], Libros de Cabildos de Lima [en adelante LCL], vol. XXVI, f. 61.

${ }^{17}$ García Bernal 2006a: 577-583.

${ }^{18}$ León Pinelo Gutiérrez s.f.: 12.

${ }^{19}$ Ib.: 5 . 
otras instituciones y gremios: «y así es bien que sirva a su relación de preludio una breve noticia de las otras». ${ }^{20}$

El impreso de la fiesta, existente en la Biblioteca Nacional de España, no tiene carátula, por lo que no se registran ni el lugar — presumiblemente Lima - ni la fecha de impresión, aunque esta última sería cercana a los hechos, como era habitual en la época. Tampoco el nombre del autor, aunque su identidad se desvela cuando, poco después de iniciar el relato, indica:

era el rector D. Diego de León Pinelo, mi padre, abogado de la Real Audiencia, protector general de los naturales deste reino por el excelentísimo señor virrey conde de Alba y catedrático de Prima de Cánones por oposición $y$ en propiedad, cuya modestia me manda que excuse otros elogios. ${ }^{21}$

Con estos datos el autor no puede ser otro que Diego de León Pinelo Gutiérrez, ${ }^{22}$ único hijo varón del entonces rector de San Marcos, Diego de León Pinelo, quien a su vez era hermano del conocido jurista Antonio de León Pinelo y del canónigo de Puebla de los Ángeles Juan de León Pinelo. ${ }^{23}$ En efecto, Juan, Antonio y Diego fueron, por este orden, los tres hijos del comerciante portugués de origen converso Diego López de Lisboa, natural de dicha ciudad. El abuelo del que sería el autor de la relación se avecindó en Valladolid, al tener que huir de su ciudad natal junto con su mujer. ${ }^{24}$ Después pasó a las Indias y se estableció primero

${ }^{20} \mathrm{Ib} .: 7$.

${ }^{21} \mathrm{Ib} .: 4$.

${ }^{22}$ Mujica (1999) indica también quién fue el autor.

${ }^{23}$ Sobre Diego de León Pinelo, véase Eguiguren 1940-1951, I: 422; Lohmann Villena 1946: 27-130; Medina 1965, I: 390-406; Torre Villar 1979: 14; y López Castillo 1996: 67-69. Sobre Antonio de León Pinelo, véase León Pinelo 1953: 220, XXV, XXXVI, XCIII-CVI, CXXXXV-CXL; Eguiguren 1940-1951, I: 427; y «Bienes de difuntos: Antonio de León Pinelo», 1664, AGI, Contratación, 971, N. 4, R. 4. Sobre Juan de León Pinelo, véase Torre Villar 1996: 15, 19, 52-61; y López Castillo 1996: 67.

${ }^{24}$ Tanto él como Catalina de Esperanza Pinelo, su mujer, descendían de judíos portugueses, algunos de los cuales fueron procesados por el Tribunal de la Inquisición en Lisboa. En Valladolid se acogieron a la protección de unos parientes religiosos, de modo especial a la del carmelita Antonio López, que gozaba de influencia en la corte (Torre Villar 1979: 11-12). Sobre la familia León Pinelo, está la tesis inédita de Pizarro Baumann (1993). 
en Buenos Aires y luego, el año 1594 o 1595, en Córdoba de Tucumán, desde donde manejó una importante red comercial con judíos holandeses y portugueses. ${ }^{25}$ Cuando su familia pasó también a las Indias, en 1604, Diego López de Lisboa quiso proporcionar a sus hijos estudios universitarios, lo cual significaba enviarlos a Lima. ${ }^{26}$

Esta digresión hacia la familia paterna del autor de la relación parece obligada por varios motivos. En primer lugar, es significativo el hecho de que los León Pinelo fueran una familia de escritores eruditos. El abuelo Diego, tras dedicarse al comercio, publicó en Lima un Epitome de la vida del ilustrísimo doctor don Fernando de Arias de Ugarte (1638). Su hijo Diego, padre del autor de la relación de fiestas, tuvo también una importante producción bibliográfica. ${ }^{27}$ De hecho, en su calidad de catedrático de San Marcos, fue autor del Hypomnema apologeticum pro regali academia limensi (1648), un opúsculo en defensa de este instituto docente que había sido omitido por el filólogo belga Justo Lipsio en su lista de universidades.

Precisamente en esta última obra, Diego elogiaba también a sus otros dos hermanos. Se refería al mayor, Juan de León Pinelo, ya fallecido entonces, como "hijo de esta academia, lleno de estudios hasta la admiración [...] de florentísimo ingenio». ${ }^{28}$ En efecto, Juan había sido el prototipo de humanista clásico y orador, además de autor de diferentes obras y prologuista de dos de las de su hermano Antonio: el Epitome y el Tratado de confirmaciones reales. ${ }^{29} \mathrm{Al}$ mencionar a este último - Antonio de León Pinelo era entonces relator del Consejo de Indias y, sin duda, la figura más descollante de la familia, tanto por su trabajo en la corte como por su enorme e interdisciplinar obra-, destacaba su «pericia de las leyes,

${ }^{25}$ Adquirió un navío para el comercio por el Río de la Plata con Brasil (Eguiguren 19401951, I: 421; López Castillo 1996: 66; León Pinelo 1953: XXX; Torre Villar 1979: 13). ${ }^{26}$ En 1622 enviudó y se trasladó a Chuquisaca, donde estudió en la Universidad con el fin de hacer carrera eclesiástica y ordenarse sacerdote. Llegó a ser mayordomo y confesor del obispo Hernando Arias de Ugarte (Medina 1965, I: 311-312).

${ }^{27}$ Ha sido recogida en Torre Villar 1979; véase también Torre Villar 1996: 40-42.

${ }^{28}$ León Pinelo 1949: 106.

29 Torre Villar 1996: 16-17. 
el conocimiento de las historias, la dulce armonía de las buenas letras, el estilo de escribir». ${ }^{30}$ Además, a ambos hermanos les unía también su afición por la poesía. Juan, siendo ya clérigo, fue nombrado juez para las composiciones escritas en castellano y portugués en el certamen poético que se celebró en San Marcos con motivo de la llegada a Lima del virrey príncipe de Esquilache, en 1615; Antonio, a su vez, fue jurado de las compuestas en lengua Latina y el secretario del concurso. ${ }^{31}$

Sin embargo, dentro de esta faceta de polígrafos de los León Pinelo, el aspecto más significativo para el tema abordado es, sin duda, la autoría de relaciones de fiestas. La primera obra impresa de Antonio fue precisamente la relación de las fiestas en honor de la Inmaculada celebradas en Lima en 1617, en la que se incluye además un poema suyo. ${ }^{32} \mathrm{Su}$ hermano Diego, por otro lado, fue autor de dos relaciones: la de exequias que se hicieron en Lima a la muerte de Felipe IV (1666) y la de la beatificación de Santa Rosa en Lima (1670). ${ }^{33}$ En la primera de las narradas por el rector, se incluía un poema de su hijo, Diego de León Pinelo Gutiérrez. ${ }^{34}$ Este último estudiaba Cánones en San Marcos y había escrito la relación de 1656 cuando tenía 20 años de edad. Aunque reconocía haber recibido una buena educación desde la infancia - de hecho, llegó a ocupar importantes puestos e incluso a ser también rector de la Universidad- ${ }^{35}$ ¿hasta qué punto no dispuso de la ayuda de su padre - quien posteriormente firmó dos relaciones de fiestas - para

\footnotetext{
${ }^{30}$ León Pinelo 1949: 134.

${ }^{31}$ Eguiguren 1940-1951, II: 759-762.

32 León Pinelo 1618; véase también Medina 1965, I: 150.

${ }^{33}$ Eguiguren 1940-1951, II: 86; León Pinelo 1666; y Medina 1965, II: 102-103.

${ }^{34}$ Nacido en Lima en 1636, obtuvo el grado en Cánones en enero de 1657 y el de doctor al ańo siguiente, cuando su padre era rector. Se ordenó sacerdote en 1660. Fue catedrático sustituto de Prima de Cánones en San Marcos y visitador del arzobispado de Los Reyes. En 1681, el arzobispo de Lima lo recomendó para una media ración en esa Iglesia haciendo referencia directa a sus méritos y a los de su padre y tío. En 1687, fue elegido rector de la Universidad de San Marcos («Informaciones: Diego de León Gutiérrez», mayo de 1672, AGI, Lima, 260, N. 7).

35 «Desde mi corta edad me [he] ocupado siempre en los estudios con mucha continuación y cuidado; y siendo cursante tuve diferentes actos lucrativos en las facultades de Cánones y Leyes, con aplauso de mis maestros y del rector del claustro y los demás que
} 
escribir la suya? Según él mismo señala, el rector estuvo muy ocupado en organizar la mascarada, pues «en su vigilancia, cuidado, noticias y erudición, libró el claustro su desempeño». ${ }^{36}$ Contara o no con ese apoyo, es indudable que el autor tuvo un privilegiado ambiente familiar que no solo lo inclinó a la literatura y la poesía sino que, además, lo vinculó desde muy joven, de manera estrecha, con la academia sanmarquina.

En ese sentido, otro aspecto a destacar en la autoría de la relación es la afinidad del narrador y su familia con la Universidad de San Marcos: los tres hermanos de la generación anterior al autor habían estudiado y enseñado en sus aulas. ${ }^{37}$ Además, Diego de León Pinelo Gutiérrez tuvo como padrino de bautismo a Sebastián Rodríguez de Mendoza, quien dos años antes había sido rector. ${ }^{38}$ Asimismo, un primo del cronista, Gregorio de Rojas y Acevedo, fue catedrático de Código en 1656 y es mencionado en la relación como el encargado de organizar el carro del colegio de San Martín. ${ }^{39}$ Tanto la cercanía de este último como la de Álvaro de Ibarra, catedrático de Prima de Leyes, casado con una sobrina de los León Pinelo, se confirman al aparecer ambos como albaceas en un testamento hecho por su padre, Diego de León Pinelo, en $1652 .{ }^{40} \mathrm{~A}$ través de ese mismo documento se puede establecer que otro hombre de confianza del rector fue Felipe de Espinosa y Mieses, regidor del Cabildo entre 1637 y $1674,{ }^{41}$ a quien pudo conocer en Valladolid o más tarde en América a través de los negocios del abuelo Diego López de Lisboa. También contaba con la amistad del padre del autor el entonces oidor de

concurrieron» («Informaciones: Diego de León Gutiérrez», mayo de 1672, AGI, Lima, 260, N. 7).

${ }^{36}$ León Pinelo Gutiérrez s.f.: 4.

37 Juan aparece en 1620 como maestro en una reunión del claustro universitario de San Marcos (Torre Villar 1996: 40-42).

38 "Informaciones: Diego de León Gutiérrez», mayo de 1672, AGI, Lima, 260, N. 7.

39 Junto con Joseph Dávila Falcó, catedrático de Vísperas (León Pinelo Gutiérrez s.f.: 5); véase también Mendiburu 2000, III: 144-145.

40 "Testamento de Diego de León Pinelo», 21 de abril de 1652, Archivo General de la Nación, Escribano: Marcelo Antonio de Figueroa, Protocolo 610; también incluido en: "Informaciones: Diego de León Gutiérrez», mayo de 1672, AGI, Lima, 260, N. 7.

${ }^{41}$ Lohmann Villena 1983, II: 124-126. 
la Audiencia de Lima Francisco Carrillo de Alderete, quien igualmente figuraba como albacea en $1652 .^{42}$ La conexión con este último —a su vez relacionado estrechamente con Felipe de Espinosa y Mieses- se remontaba a los años en que ambos habían estudiado en Salamanca.

En definitiva, se trata de un texto que responde a los cánones de las relaciones de fiestas barrocas, escrito por un criollo plenamente inserto en los círculos académicos de la ciudad de Los Reyes, con importantes conexiones dentro de la corte virreinal y un poderoso y cercano pariente y protector en la de Madrid.

\section{CALENDARIO FESTIVO: CABILDO, COMERCIANTES Y GREMIOS}

La relación de fiestas de Diego de León Pinelo Gutiérrez se centra en las celebraciones que el municipio encargó a la Universidad de San Marcos, según se ha mencionado, y solo contiene noticias puntuales de los festejos organizados por el propio Cabildo y por otras corporaciones. ${ }^{43}$ Uno de los motivos para esto último fue que el Cabildo secular había encargado su propia relación, que no se llegó a realizar. ${ }^{44}$ Así, aunque la preparada por la Universidad es la única disponible, al cotejarla con las breves referencias de Mugaburu es posible recomponer el conjunto de festejos.

El Cabildo, promotor de la solicitud, decidió publicar el edicto papal de concesión del patronazgo de la "Virgen María Nuestra Señora en su gloriosa advocación de la Limpia Concepción» el primer domingo de octubre de 1656 y comenzar entonces los festejos, que duraron más de

\footnotetext{
42 Murió en Lima en 1661 y en el testamento que dejó nombró albacea, entre otros, a Diego de León Pinelo: años antes, había sido deponente de una información de servicios del mismo, hecha en Los Reyes en 1639 (Jaeger Requejo 1985: 231-249).

43 Tras relatar brevemente las fiestas preparadas por el Cabildo entre el 14 y el 17 de octubre, el autor indica: «Cese aquí la relación de lo demás, que no es bien usurparla a mejor pluma que de propósito la escribiese y ofrece al pregón de la fama» (León Pinelo Gutiérrez s.f.: 8).

${ }^{44}$ El Cabildo de Lima encargó la descripción de las fiestas a Nicolás Flores quien, a pesar de haber recibido un adelanto de 200 pesos, no cumplió con el compromiso y tuvo que devolver dicha suma (Actas del Cabildo del 1 de diciembre de 1655, 9 de enero de 1656 y 14 de septiembre de 1657, AHML, LCL, vol. XXVI).
} 
dos meses. ${ }^{45}$ Reunidos los capitulares, lamentaron que la «estrechez de los tiempos», debida a los daños causados por el terremoto que meses antes había padecido la ciudad, no permitiera a las arcas municipales afrontar la compra de las "libreas y vestidos» que requería la ocasión. ${ }^{46}$ A pesar de ello, decidieron gastar 800 pesos de propios para celebrar la fiesta "con toda finura». ${ }^{47}$ Nombraron comisarios para los festejos a los regidores Nicolás Flores de Aguilar ${ }^{48}$ y Pedro Álvarez de Espinosa ${ }^{49}$ y les encargaron que trataran con los gremios, el Consulado y la Universidad para que cada quien acometiese las celebraciones que se estimaran oportunas. Asimismo, acordaron que la procesión organizada por el arzobispo se hiciera con "toda demostración de regocijo». ${ }^{50}$

Así, la víspera del domingo 15 de octubre de 1656, hubo por la noche «grandes fuegos» y, al día siguiente, se llevó a cabo la misa pontifical presidida por el arzobispo Pedro de Villagómez; el agustino Bartolomé Badillo predicó en ella. Por la tarde salió una procesión del convento de San Francisco hasta la catedral, en la que participaron el virrey conde de Alba de Aliste y la Audiencia, en medio del fervor popular:

se llevó en procesión [...] la Santísima Imagen de la Purísima Concepción de Nuestra Señora con diadema de sol y coturnos de Luna, ${ }^{51}$ en un trono y jardín de flores, alfombrado el suelo de rosa y panes de oro y plata. Y los balcones con ricas colgaduras y el aire con perfumes que hacían una como densa nube que

${ }^{45}$ Hasta el domingo 24 de diciembre de 1656 (Mugaburu y Mugaburu 1917, I: 42).

${ }^{46}$ El terremoto tuvo lugar en el mes de noviembre de 1655 y, aunque solo murieron dos personas, hubo numerosos dańos materiales (Mugaburu y Mugaburu 1917, I: 33-35). 47300 pesos para fuegos artificiales y 500 para toros (Acta del Cabildo del 19 de septiembre de 1656, AHML, LCL, vol. XXVI, f. 63r-v).

48 Además de poeta y escritor, fue catedrático de Vísperas de Leyes en San Marcos en 1621, asesor del Cabildo y del Tribunal del Consulado, regidor desde 1631 y, en 1637, fue elegido rector de San Marcos (Lohmann Villena 1983, II: 134-136).

${ }^{49}$ Oriundo de Santa Cruz de la Palma en Canarias, se dedicó preferentemente a la actividad comercial y se benefició también de sus dos matrimonios con criollas peruanas acaudaladas. En 1651, compró la vara de regidor de Lima (Lohmann Villena 1983, I: 29-30).

${ }^{50}$ Acta del Cabildo del 5 de octubre de 1656, AHML, LCL, vol. XXVI, f. 69r-v.

51 "coturnos de luna»: hace referencia a la iconografía inmaculista basada en el capítulo 12 del Apocalipsis «con la luna debajo de sus pies» (Real Academia Española 2014). 
exhalaba delicados olores de cazoletas, pomas, ${ }^{52}$ pastillas y pebetes ${ }^{53} \mathrm{y}$, entre ellos, el coro de religiosos partido en dos mitades cantado alabanzas a la Soberana Reina de los Cielos, que parece tiene por corona y gloria de otras glorias accidentales que se celebre con suntuosidad su Concepción Inmaculada. ${ }^{54}$

Los festejos del Cabildo se cerraron el martes 17 con una corrida de toros, habitual ya en algunas celebraciones religiosas limeñas. ${ }^{55}$ La relación se detiene a describir la lidia exaltando el valor de los criollos:

La ciudad lidió un día toros tan bravos, que solo pudieron ser trofeo de alientos españoles, rindiendo a su esfuerzo la cerviz y cólera con que entraban al circo, más por acreditarse de feroces, aunque a costa de las vidas, que confiados de que pudiesen retirar a hombres tan diestros que, a pie firme con un pequeño cuchillo, los degollaban tan instantáneamente que, embestir y morir, todo era fuerza de un resuello. ${ }^{56}$

Como era frecuente, el espectáculo del juego de cañas — con su vertiente caballeresca y de lucimiento- siguió a los toros:

[La ciudad] también jugó cañas manifestando el lucimiento, gala y brío de sus caballeros, vestidos de ricas telas con varios cambiantes de colores, airosas plumas y tan fuertes y ajustados en la silla, que al correr los caballos en su curso veloz, parejos con el aire, pareció que estaban ellos firmes en la tierra. ${ }^{57}$

El sábado siguiente, 21 de octubre, comenzaron las fiestas organizadas por el Tribunal del Consulado de Lima, que tenía a la Inmaculada por

52 «poma»: «vaso en que se queman perfumes» (Real Academia Española 2014).

53 "pebete»: "pasta hecha con polvos aromáticos, regularmente en forma de varilla, que encendida exhala un humo muy fragante» (Real Academia Española 2014).

${ }^{54}$ León Pinelo Gutiérrez s.f.: 6-7; y Mugaburu y Mugaburu 1917, I: 38.

${ }^{55}$ Acta del Cabildo del 14 de noviembre de 1656, AHML, LCL, vol. XXVI, ff. 72v-73. Mugaburu y Mugaburu confunden las fechas y sitúan el comienzo de las fiestas en noviembre y los toros, el día 22 (1917, I: 40). Sobre las corridas de toros, Sanz Serrano afirma que, aunque las hubo en las fiestas inmaculistas andaluzas celebradas entre $1613 \mathrm{y}$ 1617, no las hubo en otros lugares como Alcalá y Toledo (2008: 213). Este dato vendría a corroborar una vez más la importante conexión que existió entre la capital virreinal peruana y Sevilla.

${ }^{56}$ León Pinelo Gutiérrez s.f.: 7-8.

57 Ib.: 8 . 
patrona prácticamente desde su establecimiento. De hecho, en las ordenanzas dadas por el virrey príncipe de Esquilache se estableció otorgarle un escudo con campo azul, jarra de oro con azucenas y alrededor la leyenda "María, concebida sin pecado original». ${ }^{58}$ Esta institución representaba al grupo social de mayor poder económico debido a la importancia de la capital virreinal dentro del comercio a larga distancia con la metrópoli. La celebración comenzó con unos espectaculares fuegos artificiales, según Mugaburu los mayores que hasta entonces se había visto en la ciudad. Los seis carros de temática alegórica al misterio de la Inmaculada costaron 1000 patacones y dejaron bastantes personas quemadas «de ropa» y lastimadas. ${ }^{59}$ En la relación aparecen grandilocuentemente descritos:

El comercio enriqueció de luces la noche víspera de su día haciendo, con la invención de fuegos, luminarias y teas encendidas, que el hemisferio oscuro no necesitase de la luna ni de estrellas; el aire se llenó de suerte de relámpagos, truenos y cometas, que pareció bajaban del cielo a la plaza y no que de la tierra subían al Olimpo. ${ }^{60}$

El domingo 22 fue día "célebre y festivo", con misa pontifical en la catedral y una procesión en la que, acompañada por el virrey y la Audiencia, se sacó del convento de San Francisco la imagen de la Inmaculada, popularmente conocida como «La Chapetona». ${ }^{61}$ Por la tarde, los festejos del consulado se cerraron con la lidia de 30 toros en la plaza de Mercaderes; si se tiene en cuenta que la media de toros por corrida era 10 , es evidente que la de ese día tuvo especial relevancia. ${ }^{62}$ Después, los caballeros participaron en juegos de alcancías ${ }^{63} \mathrm{y}$, finalmente, se sirvió un

\footnotetext{
${ }^{58}$ Vargas Ugarte 1956: 136-137.

${ }^{59}$ Mugaburu y Mugaburu 1917, I: 38-39.

${ }^{60}$ León Pinelo Gutiérrez s.f.: 8.

${ }^{61}$ Es muy expresiva, en este sentido, la descripción del jesuita Cobo (1964, II: 319).

${ }^{62}$ Mugaburu y Mugaburu dan la fecha del 25 de noviembre para los toros; de nuevo, parece un error (Mugaburu y Mugaburu 1917, I: 39).

63 «jugar alcancías»: «tirárselas, corriendo montados a caballo, unos a otros, y pararlas con el escudo, donde se quebraban» (Real Academia Española 2014).
} 
«agasajo de ochenta tablas de colación» ${ }^{64}$ entre los tribunales y ministros "para el refresco de la tarde». ${ }^{65}$

El sábado 9 de diciembre, la fiesta recayó en el gremio de plateros, una corporación con alrededor de 80 tiendas abiertas en la ciudad durante el siglo XVII. ${ }^{6}$ Para demostrar su devoción a la Inmaculada, prepararon un desfile compuesto por once carros: ocho de ellos representaban jardines desde los que se iban echando flores; detrás iba uno de artificio que reproducía un galeón en el que navegaba el rey Felipe IV, representado este último por un león sobre un mundo. ${ }^{67} \mathrm{~A}$ la entrada de la plaza mayor, el galeón «hizo la salva disparando tres piezas»:

llegó desde el astillero a la plaza en pocas singladuras, donde se perdió de vista, con haber dado fondo: que las olas aun mentidas son fatales y el mar, aunque fingido, proceloso. Dieron el buen viaje sus naveleros y grumetes obedeciendo el pito y respondían los tablados, porque cierto llegó hermoso y galán a todas velas sin faltarle motón, ${ }^{68}$ escota, ${ }^{69}$ jarcia ni chafaldete, ${ }^{70} \mathrm{y}$ en todo propio desde el espolón de la proa hasta los corredores de la popa. ${ }^{71}$

El naufragio fingido del galeón fue celebrado por el público que lo asaltó y le arrebató todos sus adornos e insignias de plata:

Más al fin, como fundado en viento cayó y, cual otro Ícaro, se hizo más célebre con su ruina; y apenas le vieron así, cuando hasta los propios amigos

64 «tablas de colación»: se refiere a mesas servidas con «refacción de dulces, pastas y a veces fiambres, con que se obsequia a un huésped o se celebra algún suceso» (Real Academia Española 2014).

${ }^{65}$ León Pinelo Gutiérrez s.f.: 8; y Mugaburu y Mugaburu 1917, I: 39-41.

${ }^{66}$ En este caso, los textos de León Pinelo Gutiérrez y Mugaburu y Mugaburu corren paralelos (León Pinelo Gutiérrez s.f.: 8). Sobre los plateros, véase Ramos Sosa 1992: 296; y Durán Montero 1994: 194. En Sevilla, donde el gremio tenía gran importancia, organizaron una mascarada con siete cuadrillas en 1617 (Sanz Serrano 2008: 126-128).

${ }^{67}$ La reproducción de jardines era un artificio recurrente en la escenografía de la fiesta barroca (García Bernal 2006a: 466-469).

68 «motón»: "garrucha por donde pasan los cabos» (Real Academia Espańola 2014).

69 "escota»: «cabo que sirve para cazar las velas» (Real Academia Española 2014).

70 «chafaldete»: «cabo que sirve para cargar los puños de gavias y juanetes llevándolos al centro de sus respectivas vergas» (Real Academia Española 2014).

${ }^{71}$ León Pinelo Gutiérrez s.f.: 8-9. 
de quien se fiaba, le abordaron y, en breves horas, de toda su riqueza no le quedó flámula ni gallardete. ${ }^{72}$

La utilización del galeón como artificio festivo respondía al recurso a elementos de la vida real: en este caso, la llegada y salida de los galeones del puerto de El Callao constituían un referente cercano para los espectadores. Detrás entraron otros dos carros más «de lustre, y bien formada arquitectura con sus fachadas, arquitrabes, frisos y cornisas de plata y pintura, como lo pedía el arte». ${ }^{73}$ Mugaburu añade que en uno iba la Fama con tres ninfas sentadas; en el otro, la Virgen, representada por un ave Fénix, con muchos ángeles cantando alabanzas. ${ }^{74}$

El jueves 14 de diciembre salió de la Universidad una mascarada con seis grandes carros en la que participaron 1300 personas con diferentes disfraces; se gastaron más de 50.000 pesos. ${ }^{75} \mathrm{Tal}$ fue su éxito que el virrey Alba de Aliste dispuso que se repitiera al día siguiente. Como ya se ha mencionado, la relación de Diego de León Pinelo Gutiérrez centra su relato en la fiesta universitaria.

El 19 de diciembre, tuvo lugar la celebración de los «negros criollos» con lidia de toros y rejones. ${ }^{76}$ La noche del día 23, los gremios de herreros y sastres contrataron fuegos artificiales, en concreto «un castillo y cuatro galeras de fuego sobre cuatro juegos de carrozas muy llenas de fuego que embestían al castillo». Finalmente, el domingo 24 de diciembre, último día de las celebraciones, hubo misa pontifical y procesión dentro de la catedral. ${ }^{77}$

$72 \mathrm{Ib}$.

${ }^{73} \mathrm{Ib}$.

${ }^{74}$ No hemos podido constatar la identificación iconográfica de la Inmaculada con el ave Fénix, símbolo de regeneración espiritual: la Inmaculada lo sería por haber nacido sin pecado y por haber vencido el mal. Entendemos que se trata de una asociación puntual que apenas se utilizó (Mugaburu y Mugaburu 1917, I: 41).

${ }^{75}$ León Pinelo Gutiérrez s.f.: 6.

76 «negros criollos»: en la época se denominaba así a los que habían nacido en América.

77 Mugaburu y Mugaburu 1917, I: 42. 


\section{A MODO DE CONCLUSIÓN}

Las fiestas organizadas en honor de la Inmaculada en Lima en 1656 tienen un carácter singular por diferentes motivos. En primer lugar, según se ha señalado, las fechas no tienen como referente los ciclos festivos hispanos sino que responden a una motivación de carácter local: la concesión del patronazgo inmaculista a la ciudad por parte del papa Alejandro VII.

En segundo lugar, las fiestas de 1656 no solo se prolongaron durante más de dos meses sino que, además, contaron con la participación de las instituciones y corporaciones de poder más representativas de la ciudad, que movilizaron considerables recursos y personas. Ya se ha visto que solo la mascarada universitaria reunió a 1300 personas disfrazadas y supuso un gasto de 50.000 pesos. Las sumas de dinero libradas para festejar el acontecimiento por parte del Cabildo, el consulado y el gremio de plateros debieron ser también importantes, a juzgar por el despliegue de fastos y espectáculos.

Por último, el interés de estos eventos radica también en la existencia de la relación redactada por Diego de León Pinelo Gutiérrez, criollo de segunda generación, plenamente inmerso en algunos de los círculos de poder de la corte virreinal quien, siguiendo los parámetros propios del género, ofrecía una barroca reinterpretación de los festejos. De ahí que la relación, además de evidenciar el fervor inmaculista de la ciudad de Lima — protagonizado por el Cabildo, los gremios y la Universidad-, consiga brindar una exaltación de la propia urbe, en consonancia con la literatura corográfica de la época, que en la América hispana fue una vía de expresión y consolidación del naciente discurso criollo.

\section{BIBLIOGRAFÍA}

Bermúdez, José Manuel. 2001. Anales de la catedral de Lima: 1534 a 1824. Edición digital. Alicante: Biblioteca Virtual Miguel de Cervantes. http://www.cervantesvirtual.com/nd/ark:/59851/bmcqf8p6

Bradley, Peter T. 1992. Society, Economy and Defence in Seventeenth Century Peru: The Administration of the Count of Alba de Liste (1655-61). Liverpool: Institute of Latin American Studies, University of Liverpool. 
Cano Gutierrez, Diego. 1619. Relación de las fiestas triunfales que la insigne Universidad de Lima hizo a la Inmaculada Concepción de Nuestra Señora. Lima: Imprenta de Francisco Lasso.

Cobo, Bernabé. 1964. Obras del P. Bernabé Cobo. Edición de Francisco Mateos. Madrid: Atlas, 2 vols.

Cordón, Alicia. 1999. «Una relación de fiestas en defensa de la Inmaculada Concepción (1622)» En La Fiesta: Actas del II seminario de relaciones de sucesos ( La Coruña, 1998). La Coruña: Sociedad de Cultura Valle Inclán, 77-86.

Cortés Peña, Antonio Luis. 2003. «Andalucía y la Inmaculada Concepción en el siglo XVII». En Calderón de la Barca y la España del Barroco. Madrid: Centro de Estudios Políticos y Constitucionales, vol. I, 401-428.

Durán Montero, María Antonia. 1994. Lima en el siglo XVII: arquitectura, urbanismo y vida cotidiana. Sevilla: Diputación Provincial de Sevilla.

Eguiguren, Luis Antonio. 1940-1951. Diccionario histórico-cronológico de la Real y Pontificia Universidad de San Marcos y sus colegios: crónica e investigación. Lima: Imprenta Torres Aguirre, 3 vols.

Fernández Villanova, David. 2016. «La injerencia de las cofradías de artesanos en la organización de los oficios en Lima colonial». Investigaciones sociales. Núm. 37: 233-240.

Gámez Martín, José. 2008. «La Inmaculada Concepción, patrona de los reinos de España y Portugal. Devoción, monarquía y fiesta en la Edad Moderna». En Iberismo. Las relaciones entre España y Portugal. Historia y tiempo actual: y otros estudios sobre Extremadura. Llerena: Sociedad Extremeña de Historia, 181-194.

García Bernal, José Jaime. 2006a. El fasto público en la España de los Austrias. Sevilla: Secretariado de Publicaciones de la Universidad de Sevilla. .2006b. «Imagen y Palabra: el misterio de la Inmaculada y las solemnidades festivas en Andalucía (Siglo XVII)» En Poder y cultura festiva en la Andalucía Moderna. Córdoba: Universidad de Córdoba Servicio de Publicaciones, 79-114. Gutiérrez, Constancio. 1955. «Espańa por el dogma de la Inmaculada. La embajada a Roma de 1659 y la Bula "Sollicitudo" de Alejandro VI». Miscelánea Comillas. Vol. 13, núm. XXIV: 13-480.

Jaeger Requejo, Rafael. 1985. «Notas para la biografía del jurista peruano don García Francisco Carrillo Alderete». Revista del Archivo General de la Nación. Núm. 8: 239-261.

León Pinelo, Antonio de. 1618. Relación de las fiestas que a la Inmaculada Concepción de la Virgen N. Señora se hicieron en la real ciudad de Lima en el Perú, y principalmente de las que hizo la Congregación de la expectación del parto en la Compañia de Jesús, año 1617. Dirigida al Excelentíssimo Señor príncipe de Esquilache, virrey destos reinos. Lima: Imprenta de Francisco del Canto.

1953. El Gran Canciller de las Indias. Edición de Guillermo Lohmann Villena. Sevilla: Escuela de Estudios Hispano-Americanos. 
. 1992. Recopilación de las Indias. Edición de Ismael Sánchez Bella. México, D. F.: Escuela Libre de Derecho Miguel Ángel Porrúa, 3 vols.

León Pinelo, Diego de. 1666. Solemnidad fúnebre y exequias a la muerte del católico augustísimo rey D. Felipe Quarto el Grande N. S. que celebró en la Iglesia Metropolitana la Real Audiencia de Lima, que hoy gobierna en vacante, y mando imprimir el Real Acuerdo de Gobierno. Lima: Imprenta de Juan de Quevedo.

. 1949. Semblanza de la Universidad de San Marcos. Traducción de Luis Antonio Eguiguren. Lima: Talleres de Empresa Gráfica T. Scheuch.

León Pinelo Gutiérrez, Diego de. s.f. Fiestas que se celebraron en Lima con motivo del juramento de la pura y limpia Concepción de Nuestra Señora por la Ciudad en 1656: con la descripción de la Máscara con que cerró los regocijos la Universidad el 14 de diciembre del mismo año. [Lima]: s.e.

Lohmann Villena, Guillermo. 1946. El Conde de Lemos, virrey del Perú. Sevilla: Escuela de Estudios Hispano-Americanos.

1983. Los regidores perpetuos del cabildo de Lima (1535-1821). Crónica y estudio de un grupo de gestión. Sevilla: Exelentísima Diputación Provincial de Sevilla, 2 vols.

López Castillo, José. 1996. Antonio de León Pinelo, estudio crítico, documental y bibliográfico de su obra "El Gran Canciller de las Indias". Tesis doctoral. Madrid: Universidad Complutense de Madrid.

Medina, José Toribio. 1965. La Imprenta en Lima, 1584-1824. Ámsterdam: N. Israel, 4 vols.

Mendiburu, Manuel de. 2000. Diccionario histórico-biográfico del Perú. Parte primera: que corresponde a la época de la dominación española. Madrid: Fundación Histórica Tavera, 8 vols.

Mugaburu, Josephe de y Francisco de Mugaburu. 1917. Diario De Lima (16401694). Crónica de la época colonia. Lima: Sanmartí y Compañía, 2 vols.

Mujica Pinilla, Ramón. 1999. «Dime con quién andas y te diré quién eres: la cultura clásica en una procesión sanmarquina de 1656». En Hampe Martínez, Teodoro (editor). La tradición clásica en el Perú virreinal. Lima: Sociedad Peruana de Estudios Clásicos, 191-219.

Pizarro Baumann, Jimena. 1993. Los León Pinelo: una familia de cristianos nuevos en el Siglo XVII peruano. Tesis de Licenciatura. Lima Pontificia Universidad Católica del Perú.

Ramos Sosa, Rafael. 1992. "Los plateros de la Catedral de Lima (1614-1636)». Laboratorio de Arte. Núm. 5, tomo 1: 295-304.

Real Academa Española. 2014. Diccionario de la lengua española. Vigesimotercera edición. Madrid: Espasa.

Sanz Serrano, María Jesús. 2008. Fiestas Sevillanas de la Inmaculada Concepción en el siglo XVII: el sentido de la celebración y su repercusión exterior. Sevilla: Ayuntamiento de Sevilla. 
Torre Villar, Ernesto de la. 1979. Los pareceres de don Juan de Padilla y Diego de León Pinelo acerca de la enseñanza y buen tratamiento de los indios. México, D. F.: Universidad Nacional Autónoma de México. 1996. El Humanista Juan Rodríguez de León Pinelo. México, D. F.: Universidad Nacional Autónoma de México.

Vargas Ugarte, Rubén. 1956. Historia del culto de María en Iberoamérica y de sus imágenes y santuarios más celebrados. Madrid: Talleres Gráficos Jura, 2 vols. . 1959-1962. Historia de la Iglesia en el Perú. Burgos: Aldecoa, 5 vols.

Recepción: 10/IX/2017 Aceptación: 12/X/2017 